\title{
A REDUCTION OF THE THREE BODY PROBLEM BY MEANS OF TRANSFORMATION GROUPS
}

\author{
BY \\ JAMES W. SAUVE
}

Introduction. In Newtonian mechanics, the equations of motion of three bodies of mass $m_{i}$ and position $\left(x^{i}, y^{i}, z^{i}\right), i=1,2,3$ are given by the differential system

$$
\begin{aligned}
& \ddot{x}^{1}=m_{2}\left(x^{2}-x^{1}\right) / r_{12}+m_{3}\left(x^{3}-x^{1}\right) / r_{13}=F^{1}(x, y, z), \\
& \ddot{x}^{2}=m_{1}\left(x^{1}-x^{2}\right) / r_{12}+m_{3}\left(x^{3}-x^{2}\right) / r_{23}=F^{2}(x, y, z), \\
& \ddot{x}^{3}=m_{1}\left(x^{1}-x^{3}\right) / r_{13}+m_{2}\left(x^{2}-x^{3}\right) / r_{23}=F^{3}(x, y, z),
\end{aligned}
$$

with similar equations for $y^{i}, z^{i}, i=1,2,3$, where

$$
\begin{aligned}
r_{i j} & =\left[\left(x^{i}-x^{j}\right)^{2}+\left(y^{i}-y^{j}\right)^{2}+\left(z^{i}-z^{j}\right)^{2}\right]^{1 / 2}, \\
x & =\left(x^{1}, x^{2}, x^{3}\right), \quad y=\left(y^{1}, y^{2}, y^{3}\right), \quad z=\left(z^{1}, z^{2}, z^{3}\right), \\
\ddot{w} & =d^{2} w / d t^{2} .
\end{aligned}
$$

First integrals of this system resulting from conservation of linear momentum, angular momentum, and energy are well known. Moreover, the use of these integrals has led to a number of explicit reductions of (1.1) from order eighteen to order six. (A complete bibliography of the earlier methods of reduction can be found in [1, Chapter 2]; more recent methods are those of Whittaker [2, pp. 339-351], Wintner [3, pp. 306-319], and Wintner and van Kampen [4].)

The fact that the first integrals of linear and angular momentum are due to the invariance of a Newtonian system under the Galilean group is also well known; this invariance has been used in one form or another in most of the more recent reductions, usually in the form of contact transformations. In this paper, it will be shown that the entire reduction of (1.1) is the result of its invariance under continuous transformation groups, and also that the use of these groups will, for practical purposes, give no further reduction of the system. In order to demonstrate the equivalence of the reductions, the necessary coordinate changes will be patterned on those given by Whittaker; similar results could be obtained by following any of the classical methods.

We will begin with a brief review of the theory of continuous transformation

Received by the editors February 24, 1964 and, in revised form, August 3, 1964. 
groups as applied to differential equations. The summation convention will be used throughout.

1. Standard treatments of the theory of transformation groups can be found in [5], [6], and [7]; the application to differential equations is developed in [7, Chapter 12], [8] and [9]; [10] contains an elementary exposition for the case of two variables.

Definition 1.1. Let $G$ be a Lie group with identity element $e$, and $M^{n}$ a differentiable manifold. Let $T: M^{n} \times G \rightarrow M^{n}$ be a differentiable map from an open neighborhood of $M^{n} \times e$ (in the differentiable manifold $M^{n} \times G$ ) into $M^{n}$. Then $T$ is a local Lie transformation group (LLTG) if (1)

$$
T\left[T\left(x, g_{1}\right), g_{2}\right]=T\left(x, g_{2} g_{1}\right) \text { wherever defined; }
$$

(2) for each compact subset $K$ of $M^{n}$ there is a neighborhood $N_{K}$ of $e$ in $G$ such that, for fixed $g$ in $N_{K}$, the map

$$
T_{g}: x \rightarrow T(x, g)
$$

is a homeomorphism of $K$ onto some subset $K_{g}$ of $M^{n}$.

NoTE: We identify two such local Lie transformation groups if they coincide on some neighborhood of $M^{n} \times e$ in $M^{n} \times G$. If the map $T$ is defined, and satisfies (1.3), for all $g$ in $G$ and $x$ in $M^{n}$, then $T$ is a global Lie transformation group. In this paper all transformation groups are assumed to be local. In the case in which $G=R^{r}$ (real $r$-dimensional space), $g=\left(a^{1}, a^{2}, \cdots, a^{r}\right), a^{i}$ real, and $e=(0,0, \cdots, 0)$; in this case $T$ is called an $r$-parameter transformation group. Every such group can be generated by $r$ one-parameter groups.

Since a local transformation group is described by a Lie group, for each LLTG there is associated a unique Lie algebra $L$, called in this case an infinitesimal transformation group. $L$ is a finite-dimensional subalgebra of $L\left(M^{n}\right)$, the algebra of all differentiable vector fields on $M^{n}$. If $M^{n}$ has local coordinates $\left(t, x^{1}, x^{2}, \cdots, x^{n-1}\right)$, and $T$ is a one-parameter LLTG,

$$
\begin{aligned}
& T(t, a)=\tau(t, x, a), \\
& T\left(x^{i}, a\right)=\xi^{i}(t, x, a), \quad a \text { real, }
\end{aligned}
$$

then the corresponding Lie algebra is generated by the basis element

$$
u=h(t, x) D_{t}+f^{j}(t, x) D_{x^{j}},
$$

where

$$
\begin{aligned}
h(t, x) & =\left.D_{a} \tau(t, x, a)\right|_{a=0}, \quad f^{i}(t, x)=\left.D_{a} \xi^{i}(t, x, a)\right|_{a=0}, \\
x & =\left(x^{1}, x^{2}, \cdots, x^{n-1}\right), \quad D_{w}=\partial / \partial w .
\end{aligned}
$$

Since any $r$-parameter LLTG is generated by one-parameter groups, $L$ will always 
have a basis consisting of elements of the form (1.6). Moreover, in the neighborhood of a point in $L\left(M^{n}\right)$ at which it does not vanish, (1.6) can, by a proper change of coordinates, be reduced to the form

$$
\bar{u}=D_{\bar{x}^{n}}
$$

A LLTG $T$ can be extended to transformation groups of the associated lineelement bundle and curvature line-element bundle of $M^{n}$. The corresponding Lie algebras $L^{\prime}$ and $L^{\prime \prime}$, called respectively the first- and second-extended Lie algebras of $L$, have basis elements of the following form (corresponding to (1.6))

$$
\begin{aligned}
& u^{\prime}=h(t, x) D_{t}+f^{j}(t, x) D_{x^{j}}+g^{j}(t, x, p) D_{p^{j}}, \\
& u^{\prime \prime}=h(t, x) D_{t}+f^{j}(t, x) D_{x^{j}}+g^{j}(t, x, p) D_{p^{j}}+k^{j}(t, x, p, r) D_{r^{j}},
\end{aligned}
$$

where (indicating partial differentiation with a variable subscript)

$$
\begin{aligned}
g^{j}(t, x, p)= & \left(f_{t}^{j}-h_{t} p^{j}\right)+\left(f_{x^{k}}^{j}-h_{x^{k}} p^{j}\right) p^{k}, \\
k^{j}(t, x, p, r)= & \left(f_{x^{k} x^{m}}^{j} p^{k} p^{m}-h_{x^{k} x^{m}} p^{k} p^{m} p^{j}\right)+2\left(f_{x^{k t}}^{j}-h_{x^{k} t} p^{j}\right) p^{k} \\
& +\left(f_{t t}^{j}-h_{t t} p^{j}\right)-2\left(h_{x^{k}} p^{k}+h_{t}\right) r^{j}+\left(f_{x^{k}}^{j}-h_{x^{k}} p^{j}\right) r^{k} .
\end{aligned}
$$

Definition 1.2. Let $M^{n}$ be a differentiable manifold with local coordinates $\left(t, x^{1}, \cdots, x^{n-1}\right)$. A first-order differential equation

$$
d x / d t=F(t, x)
$$

is a cross-section from an open set $U$ of $M^{n}$ into $L\left(M^{n}\right)$. A second-order differential equation

$$
d^{2} x / d t^{2}=G(t, x, p)
$$

is a cross-section from an open set $\theta$ of $L\left(M^{n}\right)$ into $K\left(M^{n}\right)$. If $\theta=p_{1}^{-1}(U)$ (the projection map), then we say that (1.13) is defined over $U$.

Definition 1.3. A first-order differential equation is said to be invariant under a local one-parameter transformation group $T$ with infinitesimal generator $u$ if, for each diffeomorphism of an open set $U_{1}$ of $M^{n}$ onto an open set $U_{2}$ of $M^{n}$ (both contained in $U$ ) defined by $T$, the induced map $T^{\prime}$ of $L\left(\theta_{1}\right)$ onto $L\left(\theta_{2}\right)$ carries the cross-section $p=F(t, x)$ above $\theta_{1}$ onto the corresponding cross-section above $\theta_{2}$. If $T$ is given explicitly by (1.5), then (1.12) is invariant under $T$ if $d \xi \mid d \tau=F(\tau, \xi)$ for all $a$. This will be true if and only if $u^{\prime}[p-F(t, x)]=0$ when evaluated on the cross-section $p=F(t, x)$.

This definition has an obvious extension to the case of an $r$-parameter transformation group, in which case the equation $u^{\prime}[p-F(t, x)]=0$ must hold for each element of a basis for $L$. A similar definition applies to (1.13), and in this case invariance is had if and only if $u^{\prime \prime}[r-G(t, x, p)]=0$ when evaluated on the cross-section $r=G(t, x, p)$ (cf. $[9$, p. 18]). 
The following theorems, on solutions of differential equations through the use of local transformation groups, are stated only for first-order equations; any differential equation involving higher derivatives can, by addition of variables, be reduced to such a first-order system. Also, all solutions are assumed to be local: in a suitably restricted neighborhood in which both the differential equation and the LLTG are defined.

THEOREM 1.1. Let (1.12) be a first-order differential equation defined on an open subset of $R^{n}$, and suppose (1.12) is invariant under a LLTG T with Lie algebra L. If

(a) $\operatorname{dim} L=n-1$,

(b) L is a solvable Lie algebra,

(c) line elements of (1.12) are nowhere tangent to an integral curve of $T$, then solutions of (1.12) can be obtained by quadrature.

Details of the proof can be found in [7, pp. 449-451], and in [9, pp. 75-77]. The essential steps are these:

(1) A base can be chosen for $L$ in such a way that $\left(u_{1}, \cdots, u_{n-s}\right)$ is an ideal in $\left(u_{1}, \cdots, u_{n-s+1}\right), s=1, \cdots, n-2$; the corresponding LLTGT $T_{n-s}$ is normal in $T_{n-s+1}$.

(2) $u_{n}$ can be reduced to normal form $\bar{u}_{n}=D_{\bar{x}^{n}}$ by a proper change of coordinates; then $\bar{u}_{n}^{\prime}=\bar{u}_{n}$.

(3) $\bar{u}_{n}[\bar{p}-F(\bar{t}, \bar{x})]=F_{\bar{x}^{n}}\left(\bar{t}_{1}, \bar{x}\right)=0$, so that (omitting the bars in the new coordinates) (1.12) becomes

$$
\begin{aligned}
& \text { (a) } d x^{i} / d t=F^{i}\left(t, x^{1}, \cdots, x^{n-2}\right), \quad i=1, \cdots, n-2, \\
& \text { (b) } d x^{n-1} / d t=F^{n-1}\left(t, x^{1}, \cdots, x^{n-2}\right) .
\end{aligned}
$$

In this new form for the equation, (a) is a system or order $n-2$; when this has been solved, (b) can be solved by quadrature.

(4) It is not true in general that the process just described can be repeated (i.e., that $u_{n-2}$ can be reduced to normal form without reintroducing $x_{n}$ into the system); however, because of condition (b) these same steps can be applied to (1.14) (a); because of condition (a), the process can be continued with successive coordinate changes until, at the $(n-1)$ st step, the system has the form

$$
d x^{1} / d t=F^{1}(t) .
$$

This can be solved by quadrature; then, reversing the procedure, the remaining solutions can be obtained by a process which alternates coordinate changes with quadratures.

Even though the conditions of Theorem 1.1 are not satisfied, some reduction of (1.12) may be possible, as is indicated by the following theorems. 
THEOREM 1.2. Assume the condition of Theorem 1.1, except that condition (b) is replaced by

(b') $0=L_{0} \subset L_{1} \subset L_{2} \subset \cdots \subset L_{s}=L$ is a series of ideals in $L$, with $L_{i}$ maximal in $L_{i+1}$.

If $L_{i}$ has dimension $p_{i}$, then, by a change of coordinates, (1.12) can be reduced to a collection of differential equations of orders

$$
\left(p_{s}-p_{s-1}\right),\left(p_{s-1}-p_{s-2}\right), \cdots,\left(p_{2}-p_{1}\right), p_{1} \text {. }
$$

Proof. [7, pp. 451-456].

THEOREM 1.3. Assume the conditions of Theorem 1.1, except that condition (a) is replaced by

(a') $\operatorname{dim} L=r \quad(r<n-1)$.

Then (1.12) can be reduced to a system of order $n-r-1$; when this system has been solved, the remaining solutions of (1.12) can be obtained by quadrature.

Proof. The steps indicated in the proof of Theorem 1.1 may be followed without change, except that a complete reduction is not possible because of the dimension of $L$. After $r$ changes of coordinates, the LLTG has been exhausted, and the remaining system has dimension $n-r-1$.

Even though the group is not solvable, a variant of Theorem 1.3 may be used if certain special conditions are satisfied:

THEOREM 1.4. Assume that (1.12) is invariant under a LLTG T, of dimension $r$, and that $L_{0}$ is an ideal in the corresponding Lie algebra $L$, while $L-L_{0}=L_{1}$ is a subalgebra of $L$ (not necessarily an ideal). Then, if $L_{1}$ is solvable, Theorem 1.3 may be applied, using $L_{1}$ as the Lie algebra. The system remaining after this reduction is still invariant under $L_{0}$.

Proof. As pointed out in the proof of Theorem 1.1, the essential condition for a stepwise reduction is the existence of normal subgroups (or ideals in the algebra). Since $L_{1}$ is a subalgebra, it can be treated as an algebra, ignoring $L_{0}$; on the other hand, since $L_{0}$ is an ideal in $L$, any change of coordinates reducing $L_{1}$ to normal form will not affect a later reduction using $L_{0}$. This will be clear in the reduction which follows, where Theorem 1.4 will be used extensively.

2. In discussing the reduction of (1.1) by the use of Lie groups, the first task is to determine the maximal LLTG (or, equivalently, the maximum number of independent local one-parameter groups) which leave (1.1) invariant. For convenience in computation, we make the change of variables

$$
x^{i+3}=y^{i}, \quad x^{i+6}=z^{i}, \quad i=1,2,3 .
$$

A local Lie group $G$ leaves (1.1) invariant if and only if, for each element $u$ of $L$ (the Lie algebra corresponding to $G$ ), it is true that 


$$
u^{\prime \prime}\left[r^{i}-F^{i}(x)\right]=0
$$

in some neighborhood of a point $P$ on the surface $r^{i}=F^{i}(x)$ at which $u$ is defined and does not vanish. In the ten-dimensional space with coordinates $(t, x), u$ and $u^{\prime \prime}$ are given by (1.6), (1.9); note that for the system (1.1) in which $F^{2}(x)$ is independent of $p^{j}, i, j=1, \cdots, 9$,

$$
u^{\prime \prime}\left[r^{i}-F^{i}(x)\right]=k^{i}(t, x, p, r)-u\left[F^{i}(x)\right] .
$$

To determine the Lie algebra $L$ which leaves (1.1) invariant, we regard $h$ and the $f^{i}$ as unknown functions in (2.2), and replace the $F^{i}$ by their known values. From (2.3) we have immediately

$$
\begin{aligned}
& f_{x^{k} x^{m}}^{i} p^{k} p^{m}-h_{x^{k} x^{m}} p^{k} p^{m} p^{i}+2\left(f_{x^{k t}}^{i}-h_{x^{k} t} p^{i}\right) p^{k}+f_{t t}^{i}-h_{t t} p^{i} \\
& \quad-2\left(h_{x^{k}} p^{k}+h_{t}\right) r^{i}+\left(f_{x^{k}}^{i}-h_{x^{k}} p^{i}\right) r^{k}-f^{j} F_{x^{j}}^{i}=0, \quad i=1, \cdots, 9,
\end{aligned}
$$

in a suitably defined neighborhood of $P$. Substituting $F^{i}(x)$ for $r^{i}$, computing $F_{x j^{i}}^{i}$, and collecting terms as coefficients of the masses, we have

$$
M_{0}^{i}(t, x, p)+M_{1}^{i}(t, x, p) m_{1}+M_{2}^{i}(t, x, p) m_{2}+M_{3}^{i}(t, x, p) m_{3}=0, \quad i=1, \cdots, 9,
$$

where

$$
\begin{aligned}
& M_{0}^{i}=f_{x^{k} x^{m}}^{i} p^{k} p^{m}-h_{x^{k} x^{m}} p^{k} p^{m} p^{i}+2\left(f_{x^{k t}}^{i}-h_{x^{k} t} p^{i}\right) p^{k}+f_{t t}^{i}-h_{t t} p^{i}, \\
& M_{1}^{1}=\left(f_{x^{2}}^{1}-h_{x^{2}} p^{1}\right)\left(x^{1}-x^{2}\right) / r_{12}^{3}+\left(f_{x^{3}}^{1}-h_{x^{3}} p^{1}\right)\left(x^{1}-x^{3}\right) / r_{13}^{3} \\
& +\left(f_{x^{5}}^{1}-h_{x^{5}} p^{1}\right)\left(x^{4}-x^{5}\right) / r_{12}^{3}+\left(f_{x^{6}}^{1}-h_{x^{6}} p^{1}\right)\left(x^{4}-x^{6}\right) / r_{13}^{3} \\
& +\left(f_{x^{8}}^{1}-h_{x^{8}} p^{1}\right)\left(x^{7}-x^{8}\right) / r_{12}^{3}+\left(f_{x^{9}}^{1}-h_{x^{9}} p^{1}\right)\left(x^{7}-x^{9}\right) / r_{13}^{3} \text {, } \\
& M_{2}^{1}=\left[-2\left(h_{x^{k}} p^{k}+h_{t}\right)+\left(f_{x^{1}}^{1}-h_{x^{1}} p^{1}\right)\right]\left(x^{2}-x^{1}\right) / r_{12}^{3} \\
& +\left(f_{x^{3}}^{1}-h_{x^{3}} p^{1}\right)\left(x^{2}-x^{1}\right) / r_{23}^{3}+\left(f_{x^{4}}^{1}-h_{x^{4}} p^{1}\right)\left(x^{5}-x^{4}\right) / r_{12}^{3} \\
& +\left(f_{x^{6}}^{1}-h_{x^{6}} p^{1}\right)\left(x^{5}-x^{6}\right) / r_{23}^{3}+\left(f_{x^{7}}^{1}-h_{x^{7}} p^{1}\right)\left(x^{8}-x^{7}\right) / r_{12}^{3} \\
& +\left(f_{x^{9}}^{1}-h_{x^{9}} p^{1}\right)\left(x^{8}-x^{9}\right) / r_{23}^{3}+\left(f^{1}-f^{2}\right) / r_{12}^{3}+3\left(x^{2}-x^{1}\right) W / r_{12}^{5} \text {, } \\
& M_{3}^{1}=\left[-2\left(h_{x^{k}} p^{k}+h_{t}\right)+\left(f_{x^{1}}^{1}-h_{x^{1}} p^{1}\right)\right]\left(x^{3}-x^{1}\right) / r_{13}^{3} \\
& +\left(f_{x^{2}}^{1}-h_{x^{2}} p^{1}\right)\left(x^{3}-x^{2}\right) / r_{23}^{3}+\left(f_{x^{4}}^{1}-h_{x^{4}} p^{1}\right)\left(x^{6}-x^{4}\right) / r_{13}^{3} \\
& +\left(f_{x^{5}}^{1}-h_{x^{s}} p^{1}\right)\left(x^{6}-x^{5}\right) / r_{23}^{3}+\left(f_{x^{7}}^{1}-h_{x^{7}} p^{1}\right)\left(x^{9}-x^{7}\right) / r_{13}^{3} \\
& +\left(f_{x^{8}}^{1}-h_{x^{8}} p^{1}\right)\left(x^{9}-x_{x}^{8}\right) / r_{23}^{3}+\left(f^{1}-f^{3}\right) / r_{13}^{3}+3\left(x^{3}-x^{1}\right) Y / r_{13}^{5} \text {, } \\
& \text { (2.10) } W=W(f, t)=\left(f^{1}-f^{2}\right)\left(x^{1}-x^{2}\right)+\left(f^{4}-f^{5}\right)\left(x^{4}-x^{5}\right)+\left(f^{7}-f^{8}\right)\left(x^{7}-x^{8}\right) \text {, }
\end{aligned}
$$


(2.11) $Y=Y(f, t)=\left(f^{1}-f^{3}\right)\left(x^{1}-x^{3}\right)+\left(f^{4}-f^{6}\right)\left(x^{4}-x^{6}\right)+\left(f^{7}-f^{9}\right)\left(x^{7}-x^{9}\right)$, with similar expressions for $M_{k}^{j}, j=2,3, \cdots, 9 ; k=1,2,3$.

Since $m_{1}, m_{2}, m_{3}$ are nonzero constants, it follows from (2.5) that $M_{j}^{i}=0$, $i=1,2, \cdots, 9 ; j=0,1,2,3$. In particular $M_{0}^{i}=0$, so that, from (2.5) (since the $p^{i}$ are independent coordinates)
(a) $f_{t t}^{i}=0$,
(b) $f_{x^{\prime} t}^{i}=0$,
(c) $2 f_{x^{i t}}^{i}-h_{t t}=0$,
(d) $f_{x^{j} x^{k}}^{i}=0, \quad i \neq j$,
$\neq j$
(e) $f_{x^{j} x^{i}}^{i}-2 h_{x^{j} t}=0$,
(f) $h_{x^{\jmath} x^{k}}=0$,
$i, j, k=1,2, \cdots, 9$.

From (2.12), (a), (b), (d),

$$
f^{i}(t, x)=\mu^{i}\left(x^{i}\right) t+\alpha^{i}\left(x^{i}\right)+c_{j}^{i} x^{j}, \quad i, j=1,2, \cdots, 9,
$$

where $\mu^{i}$ and $\alpha^{i}$ are functions of $x^{i}$ alone, and $c_{j}^{i}$ are constants. Since $f^{i}(t, x)$ is analytic, $f_{x^{j} x^{k}}^{i}=f_{x^{k} x^{j}}^{i}$, so that, from (2.12), (d), (e),

$$
h_{x^{j_{t}}}=0 \text {, }
$$

or $h(t, x)=\lambda(t)-d_{j} x^{j}$, where the $d_{j}$ are constants. Moreover, from (e) and (2.13), $\mu_{x^{i} x^{i}}^{i}=\alpha_{x^{i} x^{i}}^{i}=0$, so that

$$
\mu^{i}=b^{i} x^{i}+c_{10}^{i} ; \quad \alpha^{i}=c_{i}^{i} x^{i}+c_{11}^{i} \quad \text { (no summation) }
$$

which, with (c), gives $2 b^{i}=\lambda_{t t}$, or

$$
\lambda=b^{i} t^{2}+d_{10} t+d_{11} \text {. }
$$

It is immediate that $b^{i}=b^{j}=b$, and we then have

$$
\begin{aligned}
& h=d_{j} x^{j}+d_{10} t+d_{11}+b t^{2}, \\
& f^{i}=c_{j}^{i} x^{j}+c_{10}^{i} t+c_{11}^{i}+b x^{i} t,
\end{aligned}
$$

where $c_{j}^{i}, d_{i}$ and $b$ are all constants, $i, j=1,2, \cdots, 9$.

Using these values for $h$ and $f^{i}$ in $M_{1}^{i}, M_{2}^{i}$, and $M_{3}^{i}$, and collecting terms as coefficients of the independent variables $x^{i}, x^{i} p^{j}$, we have, from $M_{1}^{1}$,

$$
c_{2}^{1}=c_{3}^{1}=c_{5}^{1}=c_{6}^{1}=c_{8}^{1}=c_{9}^{1}=0 ; d_{2}=d_{3}=d_{5}=d_{6}=d_{8}=d_{9}=0,
$$

while, from $M_{2}^{2}$

$$
c_{1}^{2}=c_{3}^{2}=c_{4}^{2}=c_{6}^{2}=c_{7}^{2}=c_{9}^{2}=0 ; d_{1}=d_{3}=d_{4}=d_{6}=d_{7}=d_{9}=0 .
$$

Repeating this substitution for $M_{3}^{3}, M_{1}^{4}, M_{2}^{5}, M_{3}^{6}, M_{1}^{7}, M_{2}^{8}, M_{3}^{9}$, we have

$$
\begin{aligned}
& f^{i}=c_{1}^{i} x^{1}+c_{4}^{i} x^{4}+c_{7}^{i} x^{7}+c_{10}^{i} t+c_{11}^{i}+b x^{i} t, \quad i=1,4,7, \\
& f^{j}=c_{2}^{j} x^{2}+c_{5}^{j} x^{5}+c_{8}^{j} x^{8}+c_{10}^{j} t+c_{11}^{j}+b x^{j} t, \quad j=2,5,8, \\
& f^{k}=c_{3}^{k} x^{3}+c_{6}^{k} x^{6}+c_{9}^{k} x^{9}+c_{10}^{k} t+c_{11}^{k}+b x^{k} t, \quad k=3,6,9, \\
& h=b t^{2}+d_{10} t+d_{11} .
\end{aligned}
$$


When these values are substituted into $M_{2}^{i}, M_{3}^{i}$, a similar calculation gives

$$
\begin{array}{rlrl}
b & =0, \\
c_{11}^{1} & =c_{11}^{2}=c_{11}^{3}=K_{1}, & & c_{10}^{1}=c_{10}^{2}=c_{10}^{3}=K_{4}, \\
c_{11}^{4} & =c_{11}^{5}=c_{11}^{6}=K_{2}, & c_{10}^{4}=c_{10}^{5}=c_{10}^{6}=K_{5}, \\
c_{11}^{7}=c_{11}^{8}=c_{11}^{9}=K_{3}, & c_{10}^{7}=c_{10}^{8}=c_{10}^{9}=K_{6}, \\
-c_{4}^{1}=-c_{5}^{2}=-c_{6}^{3}=c_{1}^{4}=c_{2}^{5}=c_{3}^{6}=K_{7}, \\
-c_{7}^{1}=-c_{8}^{2}=-c_{9}^{3}=c_{1}^{7}=c_{2}^{8}=c_{3}^{9}=K_{8}, \\
-c_{4}^{7}=-c_{5}^{8}=-c_{6}^{9}=c_{7}^{4}=c_{8}^{5}=c_{9}^{6}=K_{9}, \\
d_{11}=K_{10}, \quad(2 / 3) d_{10}=c_{i}^{i}=K_{11}, \quad i=1, \cdots, 9 .
\end{array}
$$

Thus we are left with eleven arbitrary constants, renamed $K_{1}, \cdots, K_{11}$. The functions $f^{i}$ and $h$ of (1.6) must then be of the following form, if the LLTG $T$ is to leave (1.1) invariant:

$$
\begin{aligned}
f^{1} & =K_{1}+K_{4} t+K_{11} x^{1}-K_{7} x^{4}-K_{8} x^{7}, \\
f^{2} & =K_{1}+K_{4} t+K_{11} x^{2}-K_{7} x^{5}-K_{8} x^{8}, \\
f^{3} & =K_{1}+K_{4} t+K_{11} x^{3}-K_{7} x^{6}-K_{8} x^{9}, \\
f^{4} & =K_{2}+K_{5} t+K_{7} x^{1}+K_{11} x^{4}+K_{9} x^{7}, \\
f^{5} & =K_{2}+K_{5} t+K_{7} x^{2}+K_{11} x^{5}+K_{9} x^{8}, \\
f^{6} & =K_{2}+K_{5} t+K_{7} x^{3}+K_{11} x^{6}+K_{9} x^{9}, \\
f^{7} & =K_{3}+K_{6} t+K_{8} x^{1}-K_{9} x^{4}+K_{11} x^{7}, \\
f^{8} & =K_{3}+K_{6} t+K_{8} x^{2}-K_{9} x^{5}+K_{11} x^{8}, \\
f^{9} & =K_{3}+K_{6} t+K_{8} x^{3}-K_{9} x^{6}+K_{11} x^{9}, \\
h & =(3 / 2) K_{11} t+K_{10} .
\end{aligned}
$$

Every element $u$ of the Lie algebra $L$ satisfying (2.23) is thus a member of the eleven-dimensional Lie algebra with the following base, obtained by letting $K_{i}=1, K_{j}=0, j \neq i, i=1, \cdots, 11$ :

$$
\begin{array}{lll}
u_{1}: & D_{x^{1}}+D_{x^{2}}+D_{x^{3}}, & u_{4}: t D_{x^{1}}+t D_{x^{2}}+t D_{x^{3}}, \\
u_{2}: & D_{x^{4}}+D_{x^{5}}+D_{x^{6}}, \quad u_{5}: t D_{x^{4}}+t D_{x^{5}}+t D_{x^{6}}, \\
u_{3}: & D_{x^{7}}+D_{x^{8}}+D_{x^{9}}, \quad u_{6}: t D_{x^{7}}+t D_{x^{8}}+t D_{x^{9}}, \\
u_{7}:-x^{4} D_{x^{1}}-x^{5} D_{x^{2}}-x^{6} D_{x^{3}}+x^{1} D_{x^{4}}+x^{2} D_{x^{5}}+x^{3} D_{x^{6}} \\
u_{8}:-x^{7} D_{x^{1}}-x^{8} D_{x^{2}}-x^{9} D_{x^{3}}+x^{1} D_{x^{7}}+x^{2} D_{x^{8}}+x^{3} D_{x^{9}} \\
u_{9}:-x^{7} D_{x^{4}}-x^{8} D_{x^{5}}-x^{9} D_{x^{6}}+x^{4} D_{x^{7}}+x^{5} D_{x^{8}}+x^{6} D_{x^{9}} \\
u_{10}: D_{t}, \quad u_{11}:(3 / 2) t D_{t}+x^{j} D_{x^{5}} \text { (summation). }
\end{array}
$$


Since condition (2.2) is necessary and sufficient, it follows that the elevenparameter group $G$ whose Lie algebra $L$ is generated by (2.24) is the largest LLTG leaving (1.1) invariant. We now wish to use this group to obtain a reduction of (1.1); to do this, it will be more convenient to return to the $(x, y, z)$ coordinates (2.1), and to write (1.1) as a first-order system

$$
\begin{aligned}
& d x^{i} / d t=p^{i}, \quad d y^{i} / d t=q^{i}, \quad d z^{i} / d t=r^{i}, \quad i=1,2,3 \\
& d p^{i} / d t=F^{i}(x, y, z), \quad d q^{i} / d t=F^{i+3}(x, y, z), \quad d r^{i} / d t=F^{i+6}(x, y, z),
\end{aligned}
$$

where the $F^{i}$ are defined as in (1.1), and the $p^{i}, q^{i}, r^{i}$ are new variables, defined by $(2.25)\left({ }^{1}\right)$. In these variables, the base for the Lie algebra becomes

$$
\begin{aligned}
& u_{1}: D_{x^{1}}+D_{x^{2}}+D_{x^{3}}, \\
& u_{2}: D_{y^{1}}+D_{y^{2}}+D_{y^{3}}, \\
& u_{3}: D_{z^{1}}+D_{z^{2}}+D_{z^{3}}, \\
& u_{4}: t D_{x^{1}}+t D_{x^{2}}+t D_{x^{3}}+D_{p^{1}}+D_{p^{2}}+D_{p^{3}}, \\
& u_{5}: t D_{y^{1}}+t D_{y^{2}}+t D_{y^{3}}+D_{q^{1}}+D_{q^{2}}+D_{q^{3}}, \\
& u_{6}: t D_{z^{1}}+t D_{z^{2}}+t D_{z^{3}}+D_{r^{1}}+D_{r^{2}}+D_{r^{3}}, \\
& u_{7}: y^{i} D_{x^{i}}-x^{i} D_{y^{i}}+q^{i} D_{p^{i}}-p^{i} D_{q^{i}} \quad \text { (summation), } \\
& u_{8}: z^{i} D_{y^{i}}-y^{i} D_{z^{i}}+r^{i} D_{q^{i}}-q^{i} D_{r^{i}}, \\
& u_{9}: x^{i} D_{z^{i}}-z^{i} D_{x^{i}}+p^{i} D_{r^{i}}-r^{i} D_{p^{i}}, \\
& u_{10}: D_{t}, \\
& u_{11}: 3 t D_{t}+2 x^{i} D_{x^{i}}+2 y^{i} D_{y^{i}}+2 z^{i} D_{z^{i}}-p^{i} D_{p^{i}}-q^{i} D_{q^{i}}-r^{i} D_{r^{i}} .
\end{aligned}
$$

Since the dimension of $L$ is less than the order of the equation (2.25), a complete solution is not possible, even locally, by the reduction theorems in $\$ 1$. Moreover $u_{7}, u_{8}$, and $u_{9}$ generate a simple three-dimensional subalgebra of $L$, so that the algebra is not solvable, and Theorem 1.3 does not apply. However, we can use Theorem 1.4. For an examination of the Lie products will show that the subalgebra $L_{1}$ generated by $u_{7}, u_{8}, u_{9}, u_{10}$, and $u_{11}$ is normal in $L$, and that the remaining base elements $u_{1}, u_{2}, u_{3}, u_{4}, u_{5}$, and $u_{6}$ generate a solvable (actually abelian) algebra $L_{2}$. Ignoring $L_{1}$, a reduction of (2.25) can be obtained using Theorem 1.3 and $L_{2}$. Once again, we assume that we are considering solutions

(1) The attempt to determine the maximal LLTG leaving (2.25) invariant leads to a differential system as difficult to solve as (2.25) itself, unless we assume that, in $u,(1.6)$, the $f^{t}$ are independent of $p^{i}, q^{i}, r^{i}$; in this case the result is identical with (2.24), or, more exactly, with (2.26), which is of the form $u^{\prime}$ (1.9). 
(or reductions) in the neighborhood of a point $P$ at which both the system (2.25) and the algebra (2.26) are defined.

The reduction using $L_{2}$ is well known: it is simply the reduction due to conservation of linear momentum. Specifically, the three basis elements $u_{1}, u_{2}$, and $u_{3}$ can be reduced to normal form (1.8)

$$
\bar{u}_{1}=D_{\bar{x}^{3}}, \quad \bar{u}_{2}=D_{\bar{y}^{3}}, \quad \bar{u}_{3}=D_{\bar{z}^{3}}
$$

by the change of coordinates which moves the origin of the space to the point $\left(x^{3}, y^{3}, z^{3}\right)$ :

$$
\begin{array}{lll}
\bar{x}^{1}=x^{1}-x^{3}, & \bar{x}^{2}=x^{2}-x^{3}, & \bar{x}^{3}=x^{3}, \\
\bar{y}^{1}=y^{1}-y^{3}, & \bar{y}^{2}=y^{2}-y^{3}, & \bar{y}^{3}=y^{3}, \\
\bar{z}^{1}=z^{1}-z^{3}, & \bar{z}^{2}=z^{2}-z^{3}, & \bar{z}^{3}=z^{3} .
\end{array}
$$

In these coordinates, the remaining base elements of $L_{2}$ become

$$
\begin{aligned}
& \bar{u}_{4}=t D_{\bar{x}^{3}}+D_{p^{1}}+D_{p^{2}}+D_{p^{3}}, \\
& \bar{u}_{5}=t D_{\bar{y}^{3}}+D_{q^{1}}+D_{q^{2}}+D_{q^{3}}, \\
& \bar{u}_{6}=t D_{\bar{z}^{3}}+D_{r^{1}}+D_{r^{2}}+D_{r^{3}} .
\end{aligned}
$$

By the change of coordinates (2.28), the variables $\bar{x}^{3}, \bar{y}^{3}, \bar{z}^{3}$ have been eliminated from (2.25); therefore the terms in $D_{\bar{x}^{3}}, D_{\bar{y}^{3}}, D_{\bar{z}^{3}}$ and their counterparts in the extended algebra play no part in the invariance of the remaining fifteenth-order equation, and may be omitted in (2.29). Then a change of coordinates similar to $(2.28)$ :

$$
\begin{array}{lll}
\bar{p}^{1}=p^{1}-p^{3}, & \bar{p}^{2}=p^{2}-p^{3}, & \bar{p}^{3}=p^{3}, \\
\bar{q}^{1}=q^{1}-q^{3}, & \bar{q}^{2}=q^{2}-q^{3}, & \bar{q}^{3}=q^{3}, \\
\bar{r}^{1}=r^{1}-r^{3}, & \bar{r}^{2}=r^{2}-r^{3}, & \bar{r}^{3}=r^{3},
\end{array}
$$

will reduce (2.29) to normal form:

$$
\overline{\bar{u}}_{4}=D_{\bar{p}^{3}}, \quad \overline{\bar{u}}_{5}=D_{\bar{q}^{3}}, \quad \overline{\bar{u}}_{6}=D_{\bar{r}^{3}},
$$

which means that the variables $\bar{p}^{3}, \bar{q}^{3}$, and $\bar{r}^{3}$ have also been eliminated, and (2.25) has been reduced to a system of order twelve, together with six other equations which can be solved by quadrature when a solution has been found for the remaining differential system. Explicitly, dropping the accents on the new variables, and omitting the equations which can be solved by quadrature,

$$
\begin{aligned}
& d x^{i} / d t=p^{i}, \quad d y^{i} / d t=q^{i}, \quad d r^{i} / d t=r^{i}, \quad i=1,2, \\
& d p^{1} / d t=F^{1}-F^{3}, \quad d q^{1} / d t=F^{4}-F^{6}, \quad d r^{1} / d t=F^{7}-F^{9}, \\
& d p^{2} / d t=F^{2}-F^{3}, \quad d q^{2} / d t=F^{5}-F^{6}, \quad d r^{2} / d t=F^{8}-F^{9},
\end{aligned}
$$


where

$$
\begin{aligned}
r_{12} & =\left[\left(x^{1}-x^{2}\right)^{2}+\left(y^{1}-y^{2}\right)^{2}+\left(z^{1}-z^{2}\right)^{2}\right]^{3 / 2}, \\
r_{13} & =\left[\left(x^{1}\right)^{2}+\left(y^{1}\right)^{2}+\left(z^{1}\right)^{2}\right]^{3 / 2} \\
r_{23} & =\left[\left(x^{2}\right)^{2}+\left(y^{2}\right)^{2}+\left(z^{2}\right)^{2}\right]^{3 / 2} \\
F^{1} & =m_{1}\left(x^{2}-x^{1}\right) / r_{12}-m_{3} x^{1} / r_{13}, \\
F^{2} & =m_{1}\left(x^{1}-x^{2}\right) / r_{12}-m x^{2} / r_{23}, \\
F^{3} & =m_{1} x^{1} / r_{13}+m_{2} x^{2} / r_{23},
\end{aligned}
$$

with similar expressions for $F^{i}, i=4, \cdots, 9$.

In order to demonstrate the equivalence of the remaining reduction to that of Whittaker, we make the further change of variables, (corresponding to a transformation to the center of mass), not strictly needed for the reduction by means of transformation groups:

$$
\begin{aligned}
& \bar{p}^{1}=m_{1} p^{1}-m_{1}\left(m_{1} p^{1}+m_{2} p^{2}\right) / m, \\
& \bar{p}^{2}=m_{2} p^{2}-m_{2}\left(m_{1} p^{1}+m_{2} p^{2}\right) / m,
\end{aligned}
$$

with similar equations for $\bar{q}^{i}, \bar{r}^{i}, i=1,2$, where $m=m_{1}+m_{2}+m_{3}$. In these coordinates, (2.32) becomes, omitting bars,

$$
\begin{aligned}
d x^{1} / d t & =\left(1 / m_{1}+1 / m_{3}\right) p^{1}+\left(1 / m_{3}\right) p^{2} \\
d x^{2} / d t & =\left(1 / m_{2}+1 / m_{3}\right) p^{2}+\left(1 / m_{3}\right) p^{1} \\
d p^{1} / d t & =-m_{1} m_{3} x^{1} / r_{13}+m_{1} m_{2}\left(x^{2}-x^{1}\right) / r_{12} \\
d p^{2} / d t & =-m_{2} m_{3} x^{2} / r_{13}+m_{1} m_{2}\left(x^{1}-x^{2}\right) / r_{12}
\end{aligned}
$$

with, again, similar expressions for $d y^{i} / d t, d q^{i} / d t, d z^{i} / d t, d r^{i} / d t, i=1,2$.

This system is still invariant under the subalgebra $L_{1}$ with basis elements $u_{7}, u_{8}, u_{9}, u_{10}, u_{11}$. A straightforward computation will show that these five basis elements have the same form (2.24) in the new coordinates (i.e., after the change of coordinates $(2.28),(2.30),(2.34))$ that they had in the original ones. Once again, the invariance of (2.35) under $L_{1}$ is not affected by omitting those terms in $D_{x^{3}}$, $D_{y^{3}}, D_{z^{3}}, D_{p^{3}}, D_{q^{3}}$, and $D_{r^{3}}$, and their corresponding terms in the extended algebra.

The basis elements $u_{7}, u_{8}, u_{9}$ generate a simple subalgebra $L_{3}$ in $L_{1}$, while $L_{4}=L_{1}-L_{3}$ is an ideal in $L_{1}$ generated by $u_{10}$ and $u_{11}$. Using Theorem 1.4 once again, we now consider only the subalgebra $L_{3}$.

Since $L_{3}$ is simple, a normalization is not possible. However, the transformation to the "invariable plane" also reduces $L_{3}$ to a desirable form. For if 


$$
\begin{aligned}
& x^{i \prime}=\left(c_{3} / d\right) x^{i}-\left(c_{1} / d\right) z^{i} \\
& y^{i \prime}=\left(-c_{1} c_{2} / c d\right) x^{i}+(d / c) y^{i}-\left(c_{2} c_{3} / c d\right) z^{i} \\
& z^{i \prime}=\left(c_{1} / c\right) x^{i}+\left(c_{2} / c\right) y^{i}+\left(c_{3} / c\right) z^{i}, \\
& p^{i \prime}=\left(c_{3} / d\right) p^{i}-\left(c_{1} / d\right) r^{i} \\
& q^{i \prime}=\left(-c_{1} c_{2} / c d\right) p^{i}+(d / c) q^{i}-\left(c_{2} c_{3} / c d\right) r^{i} \\
& r^{i \prime}=\left(c_{1} / c\right) p^{i}+\left(c_{2} / c\right) q^{i}+\left(c_{3} / c\right) r^{i}
\end{aligned}
$$

where

$$
\begin{aligned}
c_{1} & =m_{1}\left(y_{1} r_{1}-q_{1} z_{1}\right)+m_{2}\left(y_{2} r_{2}-q_{2} z_{2}\right), \\
c_{2} & =m_{1}\left(z_{1} p_{1}-x_{1} r_{1}\right)+m_{2}\left(z_{2} p_{2}-x_{2} r_{2}\right), \\
c_{3} & =m_{1}\left(x_{1} q_{1}-p_{1} y_{1}\right)+m_{2}\left(x_{2} q_{2}-p_{2} y_{2}\right), \\
c & =\left[\left(c_{1}\right)^{2}+\left(c_{2}\right)^{2}+\left(c_{3}\right)^{2}\right]^{1 / 2}, \\
d & =\left[\left(c_{1}\right)^{2}+\left(c_{3}\right)^{2}\right]^{1 / 2}
\end{aligned}
$$

then

$$
\begin{aligned}
\bar{u}_{7}= & \left(c_{3} c / d\right) y^{1 \prime} D_{x^{1}},-x^{1 \prime} D_{y^{1}}+q^{1 \prime} D_{p^{1 \prime}}-p^{1 \prime} D_{q^{1}} \\
& +y^{2 \prime} D_{x^{2}}-x^{2 \prime} D_{y^{2}}+q^{2 \prime} D_{p^{2}}-p^{2 \prime} D_{q^{2}}, \\
= & \left(c_{3} c / d^{2}\right) Y, \\
\bar{u}_{8}= & \left(c_{1} c / d^{2}\right) Y, \\
\bar{u}_{9}= & 0,
\end{aligned}
$$

where we assume $c_{1}, c_{3}, c$, and $d$ to be expressed in the new coordinates, (note that (2.35) is unchanged in form by the coordinate change (2.36)). Thus one of the three basis elements has been reduced to zero and, in fact, the other two are linearly dependent. For $d c_{1} / d t=d c_{2} / d t=d c_{3} / d t=0$, as can be shown by deriving (2.37) with respect to $t$, and using (2.35). Therefore $c_{1}, c_{2}, c_{3}, c$, and $d$ are all constant functions of $t$, and $\bar{u}_{7}$ and $\bar{u}_{8}$ differ only by a constant factor. Moreover, if in the new coordinates (2.36) we again let

$$
\bar{c}_{1}=m_{1}\left(y^{1 \prime} r^{1 \prime}-q^{1 \prime} z^{1 \prime}+y^{2 \prime} r^{2 \prime}-q^{2 \prime} z^{2 \prime}\right)
$$

with $\bar{c}_{2}, \bar{c}_{3}, \bar{c}$ and $d$ similarly derived from (2.37), a straightforward substitution will show that $\bar{c}_{1}=\bar{c}_{2}=0$, while $\bar{c}_{3}=\bar{c}=\bar{d}$, a constant.

The remaining generator $\bar{u}_{7}$ is reduced to normal form by the change of coordinates corresponding to the "elimination of the node": 


$$
\begin{array}{rlrl}
v & =\arctan a / b, & 1 & =\arctan d / c^{\prime}, \\
\xi^{i} & =\left(x^{i} b+y^{i} a\right) / d^{\prime}, & \eta^{i}=z^{i} c^{\prime} / d^{\prime}, & \\
N & =\sum_{i=1}^{2}\left[-p^{i} a\left(x^{i} b+y^{i} a\right) / d^{\prime 2}+q^{i} b\left(x^{i} b+y^{i} a\right) / d^{\prime 2}\right. \\
& \left.-c^{\prime \prime} b p^{i} z^{i} / d^{\prime 2}-a c^{\prime \prime} q^{i} z^{i} / d^{\prime 2}\right], \\
I & =\sum_{i=1}^{2}\left(1 / d^{\prime}\right)\left(p^{i} z^{i} a-q^{i} z^{i} b+r^{i} z^{i} c^{\prime \prime}\right), & i=1,2, \\
\Xi^{i} & =\left(p^{i} b+q^{i} a\right) / d^{\prime}, & \\
H^{i} & =\left(-p^{i} a c^{\prime \prime}+q^{i} b c^{\prime \prime}+r^{i} d^{\prime 2}\right) / c^{\prime} d^{\prime}, &
\end{array}
$$

where

$$
\begin{aligned}
a & =z^{1} y^{2}-z^{2} y^{1}, \quad b=z^{1} x^{2}-z^{2} x^{1}, \quad c^{\prime \prime}=x^{2} y^{1}-x^{1} y^{2}, \\
d^{\prime} & =\left(a^{2}+b^{2}\right)^{1 / 2}, \quad c^{\prime}=\left(a^{2}+b^{2}+c^{\prime \prime 2}\right)^{1 / 2}
\end{aligned}
$$

and where the accents are omitted in the old coordinates. Thus

$$
\bar{u}_{7}=-\left(c c_{3} / d^{2}\right) D_{v}=D_{v} .
$$

Moreover, (2.39) becomes, in the new coordinates (2.40),

$$
\begin{aligned}
\bar{c}_{1}= & \csc \imath \csc v \sum_{i=1}^{2}\left(\xi^{i} H^{i} \sin ^{2} v-\eta^{i} \Xi^{i} \sin ^{2} v\right) \\
& +I \sin \imath \sin v \cos v-N \sin ^{2} v \cos \imath=0, \\
\bar{c}_{2}= & \csc \imath \csc v \sum_{i=1}^{2}\left(\eta^{i} \Xi^{i}-\xi^{i} H^{i}\right) \sin v \cos v \\
& +I \sin \imath \sin ^{2} v+N \sin v \cos v \cos \imath=0, \\
\bar{c}_{3}= & N,
\end{aligned}
$$

which immediately gives

$$
\begin{aligned}
I & =0 \\
N \cos \mathrm{\imath} & =\sum_{i=1}^{2}\left(\xi^{i} H^{i}-\eta^{i} \Xi^{i}\right), \\
N & =\bar{c} \quad \text { (a constant). }
\end{aligned}
$$

Because of (2.42), the variable $v$ has been eliminated from the system, and can be obtained by quadrature; (2.44) gives values for the variables $I$, 1 , and $N$ in terms of the remaining variables. Thus, the succession of coordinate changes which reduces the simple group to a "normal" form has at the same time reduced the order of the differential equation by four. It now is of order eight, and is given explicitly by 


$$
\begin{aligned}
d \xi^{1} / d t= & \left(1 / m_{1}+1 / m_{3}\right) \Xi^{1}+\left(1 / m_{3}\right) \Xi^{2}+A \eta^{1}, \\
d \xi^{2} / d t= & \left(1 / m_{2}+1 / m_{3}\right) \Xi^{2}+\left(1 / m_{3}\right) \Xi^{1}+A \eta^{2}, \\
d \eta^{1} / d t= & \left(1 / m_{1}+1 / m_{3}\right) H^{1}+\left(1 / m_{3}\right) H^{2}-A \xi^{1}, \\
d \eta^{2} / d t= & \left(1 / m_{2}+1 / m_{3}\right) H^{2}+\left(1 / m_{3}\right) H^{1}-A \xi^{2}, \\
d \Xi^{1} / d t= & A H^{1}-B \eta^{2}-m_{1} m_{3} \xi^{1} / \rho_{1}+m_{1} m_{2}\left(\xi^{2}-\xi^{1}\right) / \rho_{12}, \\
d \Xi^{2} / d t= & A H^{2}+B \eta^{1}-m_{2} m_{3} \xi^{2} / \rho_{2}+m_{1} m_{2}\left(\xi^{1}-\xi^{2}\right) / \rho_{12}, \\
d H^{1} / d t= & -A \Xi^{1}-C\left[\left(1 / m_{2}+1 / m_{3}\right) \eta^{1}-\left(1 / m_{3}\right) \eta^{2}\right]+B \xi^{2} \\
& -m_{1} m_{3} \eta^{1} / \rho_{1}+m_{1} m_{2}\left(\eta^{2}-\eta^{1}\right) / \rho_{12}, \\
d H^{2} / d t= & -A \Xi^{2}-C\left[\left(1 / m_{1}+1 / m_{3}\right) \eta^{2}-\left(1 / m_{3}\right) \eta^{1}\right]-B \xi^{1} \\
& -m_{2} m_{3} \eta^{2} / \rho_{2}+m_{1} m_{2}\left(\eta^{1}-\eta^{2}\right) / \rho_{12},
\end{aligned}
$$

where

$$
\begin{aligned}
A & =2 R^{-2}\left[\left(1 / 2 m_{1}+1 / 2 m_{3}\right)\left(\eta^{2}\right)^{2}+\left(1 / 2 m_{2}+1 / 2 m_{3}\right)\left(\eta^{1}\right)^{2}-\eta^{1} \eta^{2} / m_{3}\right] P \\
B & =2 R^{-3}\left[\left(1 / 2 m_{1}+1 / 2 m_{3}\right)\left(\eta^{2}\right)^{2}+\left(1 / 2 m_{2}+1 / 2 m_{3}\right)\left(\eta^{1}\right)^{2}-\eta_{1} \eta^{2} / m_{3}\right]\left(N^{2}-P^{2}\right), \\
C & =-R^{-2}\left(N^{2}-P^{2}\right) \\
R & =\eta^{1} \xi^{2}-\eta^{2} \xi^{1} \\
P & =H^{1} \xi^{1}-\Xi^{1} \eta^{1}-H^{2} \xi^{2}-\Xi^{2} \eta^{2} \\
\rho_{1} & =\left[\left(\xi^{1}\right)^{2}+\left(\eta^{1}\right)^{2}\right]^{3 / 2} \\
\rho_{2} & =\left[\left(\xi^{2}\right)^{2}+\left(\eta^{2}\right)^{2}\right]^{3 / 2} \\
\rho_{12} & =\left[\left(\xi^{1}-\xi^{2}\right)^{2}+\left(\eta^{1}-\eta^{2}\right)^{2}\right]^{3 / 2}
\end{aligned}
$$

There still remains the two-parameter LLTG whose algebra $L_{4}$ has as basis $u_{10}$ and $u_{11}$. After the changes of coordinates (2.36), (2.40), these two basis elements have the following form:

$$
\begin{aligned}
& u_{10}=D_{t}, \\
& u_{11}=3 t D_{t}+N D_{N}+I D_{I}+\sum_{i=1}^{2}\left(2 \xi_{\xi^{i}}^{i D}+2 \eta_{\eta^{i}}^{i_{D}}-H_{H^{i}}^{i_{D}}-\Xi_{\Xi^{i}}^{i_{D}}\right) .
\end{aligned}
$$

The system (2.45) is no longer invariant under this two-parameter group since, by introducing the constant $N$, it is no longer homogeneous, and does not admit $u_{11}$. However, if we consider $N$ as a variable for the moment (so that the system is of order nine, with the added equation

$$
d N / d t=0
$$


which is again homogeneous), then this new system is invariant under the group with Lie algebra generated by (2.47).

Since the algebra $L_{4}$ is solvable, Theorem 1.3 can be used to obtain the final reduction. Of the two basis elements, $u_{10}$ is already in normal form, and indicates only that the differential equation is autonomous. So far as this author knows, $u_{11}$ (or the corresponding one-parameter group) has never been used in the reduction of the three body problem. If the reduction by the use of transformation groups is to reflect the classical reductions, then $u_{11}$ must correspond to the conservation of energy; but there is no apparent reason why this should be so, especially since $u_{11}$ has time $(t)$ as an explicit variable, and, on the other hand, conservation of energy does not demand homogeneity. However, the use of the energy constant, given in terms of the original variables by

$$
\begin{aligned}
& h=(1 / 2) \sum_{j=1}^{3}\left[\left(p^{j}\right)^{2}+\left(q^{j}\right)^{2}+\left(r^{j}\right)^{2}\right] / m_{j}-m_{2} m_{3} / r^{\prime}{ }_{23} \\
&-m_{1} m_{3} / r^{\prime}{ }_{13}-m_{1} m_{2} / r_{12}^{\prime},
\end{aligned}
$$

(where $\left.r_{i j}^{\prime}=\left[\left(x^{i}-x^{j}\right)^{2}+\left(y^{i}-y^{j}\right)^{2}+\left(z^{i}-z^{j}\right)^{2}\right]^{1 / 2}\right)$, does reduce $u_{11}$ to normal form. For if

$$
\begin{aligned}
& t^{\prime}=t h^{3 / 2}, \\
& \eta^{2 \prime}=\eta^{2} h, \\
& \xi^{1 \prime}=-1 / 2(\log h) \xi^{1}, \\
& \Xi^{i \prime}=\left(\Xi^{i}\right)^{-2} h, \quad i=1,2, \\
& \xi^{2 \prime}=\xi^{2} h, \\
& H^{i \prime}=\left(H^{i}\right)^{-2} h, \quad i=1,2, \\
& \eta^{1 \prime}=\eta^{1} h, \\
& N^{\prime}=N^{2} h \text {, }
\end{aligned}
$$

then, in these new variables,

$$
\bar{u}_{11}=D_{\xi^{1}}, \quad \bar{u}_{10}=h^{-3 / 2} D_{t} .
$$

The normal form of $\bar{u}_{11}$ shows that $\xi^{1}$ has been eliminated, and the system reduced to order eight. Since both $N$ and $h$ are both constants, $N^{\prime}$ is a constant, which reduces the system to order seven. Finally, since $h$ is a constant, $\bar{u}_{10}$ is still in normal form and can be used to reduce the system to order six, a reduction which will not be given explicitly.

We are thus able, by the use of the eleven-parameter Lie group whose Lie algebra has as basis (2.24), to obtain a reduction of the differential equation (1.1) from order eighteen to order six, which corresponds exactly with the classical reductions. Since (2.24) is maximal, no further reduction is possible by this method.

The author would like to thank Daniel C. Lewis, Jr. for his help in the preparation of this paper, and the referee for his many helpful suggestions leading to its revision. 


\section{REFERENCES}

1. R. Marcolongo, Il problema dei tre corpi, Ulrice Hoepli, Milan, 1919.

2. E. T. Whittaker, Analytical dynamics of particles and rigid bodies, 4 th ed., Cambridge, Univ. Press, Cambridge, 1960.

3. Aurel Wintner, The analytical foundations of celestial mechanics, Princeton Univ. Press, Princeton, N. J., 1947.

4. A. Wintner, and E. R. van Kampen, On a symmetrical canonical reduction, Amer. J. Math. 59 (1937), 153-166.

5. L. Pontrjagin, Topological groups, Princeton Univ. Press, Princeton, N. J., 1939.

6. Claude Chevally, Theory of Lie groups, Princeton Univ. Press, Princeton, N. J., 1946.

7. Luigi Bianchi, Lezioni sulla teoria dei gruppi continui finiti di transformazioni, Pisa, 1918.

8. S. Lie and G. Scheffers, Vorlesungen über Differentialgleichungen mit Bekanten Infinitesimalen Transformationen, Teubner, Leipzig, 1891.

9. Lawrence Markus, Group theory and differential equations, (Lecture Notes), University of Minnesota, Minneapolis, Minn., 1959-1960.

10. Abraham Cohen, An introduction to the Lie theory of one-parameter groups, Stechert, New York, 1931. (Original publication, 1911).

\section{WoOdsTOCK COLlEGE,}

WOODSTOCK, MARYLAND 